\title{
ERRATUM
}

Open Access

\section{Erratum to: Senna alata leaf extract restores insulin sensitivity in high-fat diet-induced obese mice}

Jarinyaporn Naowaboot ${ }^{1 *}$ and Pritsana Piyabhan ${ }^{2}$

\section{Erratum}

Unfortunately, after publication of the original version of this article [1] the author noticed an error. In Table 1 result (mcp-1), the symbolic significance of senna groups should be ${ }^{* * *}$ rather than *. Please find the correct Table 1 below.

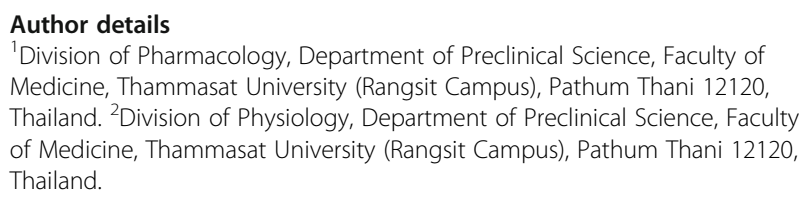

${ }^{1}$ Division of Pharmacology, Department of Preclinical Science, Faculty of Medicine, Thammasat University (Rangsit Campus), Pathum Thani 12120, Thailand. ${ }^{2}$ Division of Physiology, Department of Preclinical Science, Faculty of Medicine, Thammasat University (Rangsit Campus), Pathum Thani 12120, Thailand.

Received: 22 September 2016 Accepted: 22 September 2016 Published online: 05 October 2016

\section{Reference}

1. Naowaboot J, Piyabhan P. Senna alata leaf extract restores insulin sensitivity in high-fat diet-induced obese mice. Clinical Phytoscience. 2016;2:18.

* Correspondence: naowaboot@yahoo.com

${ }^{1}$ Division of Pharmacology, Department of Preclinical Science, Faculty of Medicine, Thammasat University (Rangsit Campus), Pathum Thani 12120, Thailand 
Table 1 Effect of S. alata leaf extract on metabolic parameters in HFD-induced obese mice

\begin{tabular}{|c|c|c|c|c|}
\hline \multirow[t]{2}{*}{ Groups } & \multirow[t]{2}{*}{ NC } & \multirow[t]{2}{*}{$\mathrm{OB}$} & \multicolumn{2}{|c|}{$\mathrm{OB}+$ S. alata $(\mathrm{mg} / \mathrm{kg})$} \\
\hline & & & 250 & 500 \\
\hline Body weight (g) & $44.0 \pm 0.70$ & $50.0 \pm 1.68^{*}$ & $48.6 \pm 1.20^{*}$ & $48.7 \pm 1.13^{*}$ \\
\hline FBG (mM) & $5.5 \pm 0.14$ & $9.9 \pm 0.61^{*}$ & $7.5 \pm 0.28^{*, * *}$ & $7.4 \pm 0.16^{*}, * *$ \\
\hline Insulin (ng/mL) & $0.6 \pm 0.06$ & $3.6 \pm 0.23^{*}$ & $2.4 \pm 0.26^{*, * *}$ & $2.3 \pm 0.25^{* * * *}$ \\
\hline HOMA-IR & $3.8 \pm 0.41$ & $37.7 \pm 3.08^{*}$ & $19.2 \pm 1.84^{* * * *}$ & $18.7 \pm 2.96^{*, * *}$ \\
\hline Leptin (ng/mL) & $1.3 \pm 0.28$ & $15.0 \pm 1.15^{*}$ & $10.3 \pm 0.72^{*}, * *$ & $10.0 \pm 0.72^{*}, * *$ \\
\hline Adiponectin (pg/mL) & $7.6 \pm 0.25$ & $5.9 \pm 0.44^{*}$ & $9.1 \pm 0.31^{*},{ }^{* *}$ & $9.6 \pm 0.26^{*, * *}$ \\
\hline MCP-1 (pg/mL) & $3.9 \pm 0.46$ & $16.2 \pm 1.10^{*}$ & $5.0 \pm 0.67^{* *}$ & $3.9 \pm 0.50^{* *}$ \\
\hline TNF-a (pg/mL) & $5.1 \pm 0.70$ & $18.5 \pm 1.52^{*}$ & $10.6 \pm 0.72^{*, * *}$ & $8.7 \pm 0.61^{* * * *}$ \\
\hline
\end{tabular}

Values are represented as mean \pm SEM $(n=8) .^{*} P<0.05$ compared to normal control group

${ }^{* *} P<0.05$ compared to obese control group. NC normal control mice fed with LFD, OB obese mice fed with HFD, FBG fasting blood glucose, MCP-1 monocyte chemoattractant protein-1, TNF- $a$ tumor necrosis factor- $a$ 